\title{
Atomic ordering and coercive force of nanocrystalline Fe-Co alloy films
}

\author{
Nataliya Repina \\ Krasnoyarsk Institute of Railway Engineering, 89, L.Ketskhoveli St, Krasnoyarsk, 660028, Russia
}

\begin{abstract}
In single crystal films of the $\mathrm{Fe}_{0,5} \mathrm{Co}_{0,5}$ alloys grown by the method of vacuum condensation, a metastable condition may be received that is similar to the completely disordering condition. Approximately during ten days after the condensation in the film at the room temperature the short range and then long range atomic ordering is developed. Simultaneously the changes of magnetic anisotropy, electroresistance and coercive force of the films were investigated. This investigations show that the appearance of the shot range atomic ordering increases the electroresistance of films and the long range atomic ordering reduces it. The dependence of coercive force from time to time at room temperature atomic ordering films $\mathrm{Fe}_{0,5} \mathrm{Co}_{0,5}$ alloys, and $\mathrm{Fe}_{0,75} \mathrm{Co}_{0,25}$. Found that the biggest change Hc (double) is observed in single-crystal films when changing mechanism of magnetization reverse. Most is a consequence of the changes to the crystallographic anisotropy.
\end{abstract}

Iron-cobalt alloys, possessing interesting magnetic properties have been long attracting the researcher's attention.

It is known that the processes of atomic ordering in ferromagnetic alloys with the high Curie temperature, presenting the phase transition of the second kind, proceeds comparatively fast.

The speed of temper necessary for the suppress of the ordering condition in stechiometrical composition $\mathrm{Fe}-\mathrm{Co}$ alloy is $6000^{\circ} / \mathrm{s}$. Therefore it is very difficult to receive a disordering condition of alloy $\mathrm{Fe}_{0,5} \mathrm{Co}_{0,5}$ in massive specimens.

In the given work the processes taking place in epitaxial magnetic films at the room temperature were investigated. The changes of magnetic anisotropy, electroresistance and coercive force at natural aging were observed.

Single (001) films $\mathrm{Fe}_{0,5} \mathrm{Co}_{0,5}$ and $\mathrm{Fe}_{0,75} \mathrm{Co}_{0,25}$ were grown by the method of vacuum condensation on the surfaces of the splits of $\mathrm{LiF}$ and $\mathrm{MgO}$ crystals heating to $150^{\circ} \mathrm{C}$. In the just condensed films, having the body-centered cubic (bcc) structure, showed the absence of the super-structure phase. If you store the tapes at room temperature, i.e. as a result of natural aging.

The thickness of research films was laid in the interval 30-50 nm.

Polycrystalline iron-cobalt alloy films received on glass substrates.

Magnetic anisotropy was measured by the method of the revolving moments. Easy axis of magnetization these films depending on alloy composition are guided either along the underlying sample plane crystallographic directions $[100]\left(\mathrm{K}_{1}>0\right)$ or along the $[110]$ directions $\left(\mathrm{K}_{1}<0\right)$. The relative error of measurement of $\mathrm{K}_{1}$ is $7.5 \%$.

The character and the value of the tensions in the film specimens were determined by the X-ray diffractometrical method. The chemical composition was studied with the aid of the rentgenospectral fluorescent method.

According to these observations and the structure's investigations it was shown that the alloy $\mathrm{Fe}_{0,5} \mathrm{Co}_{0,5}$ may be received in the disordering condition comparatively easily in the thin magnetic films. The process of atomic ordering in these films may take place at room temperature and even at much lower temperatures.

As reported in the papers [1, 2], Fe-Co alloy films, under the process conditions are disordering and ordered then at room temperature.

To study the ordering in the alloys it was used «the method of the small makeweights», theoretic proposed by Krivoglas and experimentally was confirmed by us [3]. Au up to 1 at. $\%$ and $P d$ up to $1-3$ at. \% was used as makeweights.

The electroresistance of films was measured by the potenciometrical method.

The changes of elecroresistance of single crystal films $\mathrm{Fe}_{0,5} \mathrm{Co}_{0,5}$ take place at natural aging (for 10 days). The results of the measuring electroresistance $\left(\frac{\Delta \rho}{\rho}\right)$ are shown in fig.1.

It can be seen in fig. 1 that the most changes take place during the first three days. The electroresistance of 
films continue to increase until the sixth day. Then it begins to decrease slowly to the tenth day. After that the quantity of electroresistance is stabilized.

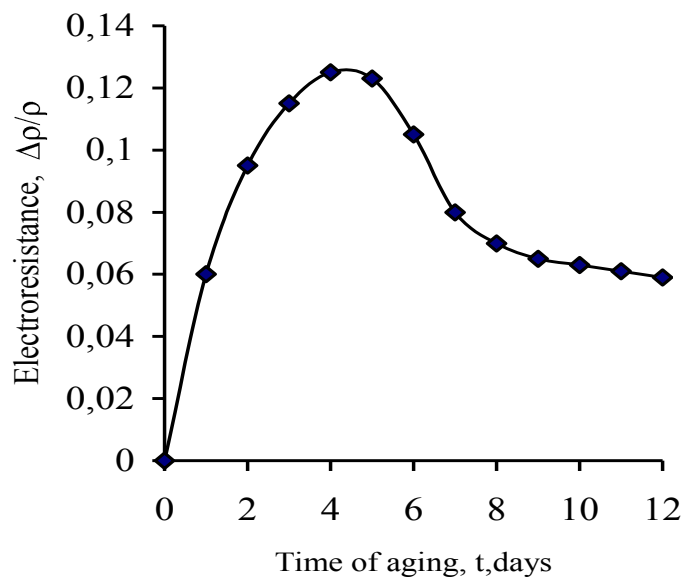

Fig.1. The changes electroresistance $\Delta \rho / \rho$ of the nanocrystalline films $\mathrm{Fe}_{0,5} \mathrm{Co}_{0,5}$ during the natural aging

Because of the coercive force $\mathrm{H}_{\mathrm{c}}$ of ferromagnetic being a structurally sensitive characteristic it aroused our interest to trace the changes $\mathrm{H}_{\mathrm{C}}$ of single crystal films $\mathrm{Fe}-\mathrm{Co}$ alloys during the time at the room temperature. The coercive force of films was defined from the magnetic hysteresis loops, with the help of magnetooptical Kerr effect. In fig. 2 the dependence of $\mathrm{H}_{\mathrm{C}}$ the films on the aging time of equiatomic composition $\mathrm{Fe}-\mathrm{Co}$ alloy is depicted.

The coercive force was measured under the magnetization reversal of films along one of the easy magnetic axis. The relative error of measurement of $\mathrm{H}_{\mathrm{C}}$ is $5 \%$.

The figures show that the changes of $\mathrm{H}_{\mathrm{C}}$ were absent during the first three days. After three days of aging the coercive force of film begins to decrease slowly. Between the seventh and tenth days of aging the sharp (approximately 2 times) decrease of $\mathrm{H}_{\mathrm{C}}$ was observed.

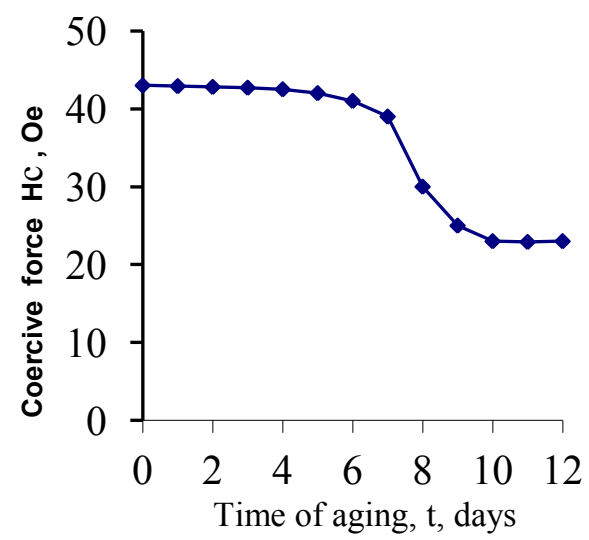

Fig.2. The changes $\mathrm{Hc}$ of nanocrystalline films $\mathrm{Fe}_{0,5} \mathrm{Co}_{0,5}$ during the natural aging
In the course of the investigation of magnetocrystalline anisotropy stechiometrical compositions the epitaxial films it was discovered that the first constant of this anisotropy changes its value from $-5 \times 10^{5}$ to $-0,5 \times 10^{5} \mathrm{erg} / \mathrm{sm}^{3}$. The changes of $\mathrm{K}_{1}$ are insignificant during three days. The principal changes of $\mathrm{K}_{1}$ films begin for three days and they finish for nine days. The energy of activation process of the change $\mathrm{K}_{1}$ process during the natural aging $(\mathrm{E}=0,25 \mathrm{eV})$ was measured. The change of $\mathrm{K}_{1}$ films at natural aging are similar to the change of $\mathrm{K}_{1}$ at ordering annealing of massive specimens.

The electronographical investigations of the films were conducted with the application of «the method of the small makeweights». 0, $4 \%$ Au were used as makeweights. In the just condensed films, having the body-centered cubic (bcc) structure, showed the absence of the super-structure phase (fig.3 a). The diffuse background on the electronograms of films appears after two day, (fig. 3 b), which is intensified during the time (fig.3 c), the electronogram took off six day), which is intensified during the time.

On the eighth day the weak reflexes type $\{100\}$ appears (fig. 3 d), and a diffuse background on the electronograms of films begins to weaken, however it does not disappear completely.

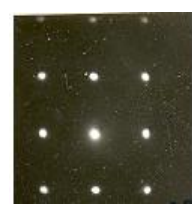

a)

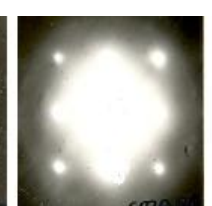

b)

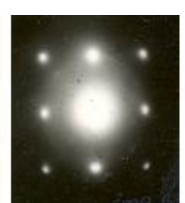

c)

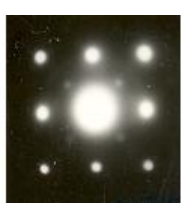

d)
Fig.3. Electronograms of single magnetic films . $\mathrm{Fe}_{0,5} \mathrm{Co}_{0,5}$ :

a) at once after the condensation of film;

b) at three days of the natural aging;

c) at six days of the natural aging;

d) at ten days of the natural aging

The electronomicroscopical investigations of films show that just after condensation they are homogeneous in structure. During the time the inhomogeneous structure appears along the edges of the grains which then turn into pores, disorderly distributed on the film, the number and size of which increases during the time.

In the electronomicroscopical photographs of films vacancy's loops appear along the edges of the grains.

The formation of the film in greatly unequilibrium conditions of the vacuum condensation leads to the saturation defects of the «vacancy» type. At the natural aging of the films the transfer of the vacancies, take place the formation of the accumulations, to slam of vacancy discs and the formation of pores take place too. It is confirmed accumulations, to slam of vacancy discs and accumulations, to slam of vacancy discs and the formation of pores take place too. It is confirmed well with the electronografical and electronomicroscopic investigations. 
The appearance of the strong diffuse background on the electronograms of the films during the first days of aging of the films can be explained by two reasons: the formation of vacancy accumulations and the formation of the short-range ordering.

The presence of reflexes $\{100\}$ is connected with the formation of the super-structure type B2.

It is obvious that the presence of the great quantity of vacancies facilitates the process of atomic ordering. The quantity of energy activation of change $\mathrm{K}_{1}(\mathrm{E}=0,25 \mathrm{eV})$ corresponds to energy the movement of vacancies.

The above described changes of electroreistance films at natural aging can be explained by the appearance of the short-range and long-range atomic ordering. The formation of the short-order in film and the appearance such inhomogeneous structure of crystal construction such as the vacancies loops and pores lead to the increase the electroresistance. $\left(\frac{\Delta \rho}{\rho}\right)$ increases to the appearance of superstructure reflexes. Since this moment the electroresistance begin to decrease, that can be explained by the increase of the degree of the long range atomic ordering. The measure of $\left(\frac{\Delta \rho}{\rho}\right)$ after ten days of aging of the film does not fall under the original measure of $\left(\frac{\Delta \rho}{\rho}\right)$ in the film that is connected with the presence comparatively great defects (pores, vacancy loops and disks) [4].

The investigations show that process of atomic ordering influences on the coercive force of single crystalline films weakly. The observed sharp decrease of $\mathrm{H}_{\mathrm{c}}$ on the 7-8 days of aging can be explained by the change of the process magnetization reversal of the film, connected with the decrease of the magnetocrystalline anisotropy. With the high measure $\mathrm{K}_{1}$ of the film was reversely magnetized by the formation of the wedge manner domains of $90^{\circ}-$ neighbouring, orientated along hard magnetic axis and by the removal of domain walls.

This magnetization reversal is typical for two axis films. When the measure $\left|K_{1}\right|$ becomes little, the film is reversed as uniaxial, that is by the formation and growth of the wedges of $180^{\circ}$-neighbouring.

According to the theory of Kondorsky [5] the field sufficient for the domain-wall movement is determined with the expression:

$$
H_{C}=\frac{P}{I_{S}}\left(\operatorname{Cos} \vartheta_{1}-\operatorname{Cos} \vartheta_{2}\right)^{-1},
$$

where P-constant; determined by the structural inhomogeneous and the strains of the material; $I_{s}-$ saturation magnetization of material; $\theta_{1}$ and $\theta_{2}$ - angles between the direction a field and the magnetization vectors on both sides of the domain wall.

In case of the magnetization reversal with $180^{\circ}$ domain-wall movement according to this formula the coercive force must be 2 times less that under the magnetization reversal $90^{\circ}$ domain-wall movement.

For polycrystalline films alloys $\mathrm{Fe}_{0,5} \mathrm{Co}_{0,5}$ и and $\mathrm{Fe}_{0,75} \mathrm{Co}_{0,25}$ studied the dependence of coercive force from the time of natural aging. Growth of coercive force alloy films $\mathrm{Fe}_{0,75} \mathrm{Co}_{0,25}$ due to an increase in anisotropy of individual crystallites films, and, therefore, the variance of the anisotropy of the film as a whole. For polycrystalline films alloy $\mathrm{Fe}_{0,5} \mathrm{Co}_{0,5}$ increase driving order, on the contrary, by lower anisotropy of individual crystallites.

Obtained in this study results indicate great opportunities offered by the practice of magnetic phenomena research samples of alloys, Atomic ordering in which occurs at room temperature. In particular, on the basis of the observed changes when you change the coercive force of the mechanism of magnetization reverse films, it can be concluded that the change in the mechanism of magnetization reverse sample can substantially alter the loss for hysteresis (in duplicate).

The results of above described the conclusion that in film specimens $\mathrm{Fe}-\mathrm{Co}$ may be received in the disordering condition comparatively easily. This condition of alloy is metastable and at the room temperature it takes place gradually in the atomic ordering condition. These data facilitate the study of atomic ordering influence on the physical properties of alloy.

\section{References}

1. N.M. Repina, Izvestiya RAN. Seriya fizicheskaya, 77, 3 . (2013)

2. N.M. Repina, Bull. R: Phys. Acad. Sci. Physics, 77, 3 (2013)

3. A.S. Komalov, N.M. Repina: Fiz. Met. Met. .37 (1974)

4. N. Hayashi, F. Stromberg and W. Keune, T. Toriyama and M. Yamashiro, I. Sakamoto: J. Appl. Phys. 101 (2007).

5. E.I. Kondorsky, J.E.T.P, 7 (1937) 